\title{
Variabilité écophysiologique et enzymatique de Cydia pomonella L en fonction de l'origine géographique et de la plante hôte
}

\author{
R Buès ${ }^{\star}$, JF Toubon, HS Poitout \\ INRA, station de zoologie, domaine Saint-Paul, site Agroparc, F-84914 Avignon cedex 9, France
}

(Reçu le 20 octobre 1994 ; accepté le 24 mai 1995)

\begin{abstract}
Résumé - Plusieurs caractères écophysiologiques et enzymatiques ont été étudiés chez des populations de Cydia pomonella, prélevées dans des localités géographiquement éloignées et sur des plantes hôtes différentes (pommier, poirier, cognassier, noyer, abricotier). Des seuils photopériodiques d'induction de la diapause et thermiques de réactivation post-diapause différents sont observés entre localités mais non entre plantes hôtes. L'analyse enzymatique de 7 loci polymorphes ne fait apparaître qu'une faible différenciation génétique, aussi bien entre les échantillons issus de localités différentes $(F s t=0,0294)$ qu'entre ceux prélevés sur les diverses plantes hôtes dans une même localité $(F s t=$ 0,0385 ). On observe cependant, entre localités, des différences significatives de fréquences alléliques. L'hypothèse est avancée de populations locales, essentiellement inféodées au pommier, dont une partie émigre, plus ou moins régulièrement, vers des hôtes plus occasionnels, en fonction de coïncidences spatio-temporelles. Toutefois, cette dispersion des adultes ne peut constituer un "effet fondateur" que temporaire, car l'arrêt de développement hivernal et le décalage des stades phénologiques sensibles des plantes hôtes concourent au brassage régulier des sous-populations.
\end{abstract}

diapause / électrophorèse / variabilité géographique / plante hôte / flux génique

Summary - Ecophysiological and enzymatic variability of Cydia pomonella L according to geographical origin and host plant. Simultaneous ecophysiological and enzymatic investigations were performed on Cydia pomonella populations from distant areas and from various host plants (apple, pear, quince, walnut and apricot). Different photoperiodic thresholds for diapause induction and temperature thresholds for post-diapause reactivation were observed according to areas but not host plants. The enzymatic analysis of 7 polymorphic loci exhibits only a weak genetic differentiation between populations from different localities $\left(F_{s t}=0.0294\right)$ and different host plants $\left(F_{s t}=0.0385\right)$. However, significant differences of allelic frequencies were noted. We put forward the hypothesis of local populations, mostly apple pests, some of which emigrate to occasional hosts, according to spatio-temporal synchronisms. Nonetheless, this dispersion does not constitute a 'foundation effect', since arrested winter development and the non-coincidence of the sensitive phenological stages of host-plants lead to a regular mixing of subpopulations.

diapause / electrophoresis / geographic variability / host plant / gene flow

* Correspondance et tirés à part 


\section{INTRODUCTION}

Dans toute son aire de répartition géographique, le carpocapse des pommes (Cydia pomonella $\mathrm{L}$ ) peut être observé sur différentes plantes hôtes (Bovey, 1966). L'influence de l'alimentation sur le développement, l'induction et l'intensité de la diapause, et en conséquence sur le voltinisme de l'espèce, a été montrée par Phillips et Barnes (1975). D'autres travaux font état du rôle de la température et de la. photopériode sur la synchronisation du cycle évolutif de l'espèce avec les conditions du milieu (Shel'Deshova, 1967 ; Rield et Croft, 1978). On peut donc formuler l'hypothèse que, sous l'action conjointe d'un décalage des stades réceptifs de différentes plantes hôtes aux attaques et d'une pression de sélection exercée notamment par les conditions abiotiques du milieu, des populations puissent se différencier génétiquement, plus ou moins selon l'importance des flux géniques. Une première comparaison du polymorphisme enzymatique chez plusieurs populations géographiquement éloignées de $C$ pomonella, prélevées uniquement sur pommier (Buès et Toubon, 1992), fait cependant apparaître une faible hétérogénéité génétique. Dans cette étude, nous poursuivons la comparaison, sur des critères écophysiologiques (seuils photo- périodiques d'induction de la diapause et thermiques de réactivation du développement postdiapause), de plusieurs échantillons de ces populations d'origines géographiques différentes. Parallèlement, nous analysons le polymorphisme enzymatique d'échantillons prélevés sur différentes plantes hôtes, dans différentes localités. Les résultats conduisent à une meilleure compréhension de la structure génétique des populations et des flux géniques qui existent entre elles.

\section{MATÉRIEL ET MÉTHODES}

\section{Échantillonnages}

Pour les 2 types d'études, les populations ont été prélevées soit dans les fruits, soit dans des bandes pièges disposées sur le tronc des arbres fruitiers. Les expérimentations d'écophysiologie ont eu lieu en $1^{\text {re }}$ génération d'élevage et les analyses enzymatiques sur les populations naturelles. Les localités, les hôtes et les dates de récoltes sont indiquées dans le tableau $\mathrm{I}$. Pour l'analyse enzymatique, seuls les échantillons 1 à 12, provenant de différentes localités et plantes hôtes, ont été analysés. Les échantillons 13 à 19 n'ont été utilisés que pour les études d'écophysiologie (les résultats de leur analyse enzymatique sont indiqués

Tableau I. Origine géographique (localités, départements ou pays), plante hôte et année d'émergence des différents échantillons de populations.

\begin{tabular}{|c|c|c|c|c|}
\hline$N^{o}$ & Origine & & Plante hôte & Année \\
\hline 1 & Avignon & (Vaucluse) & Poirier & 1991 \\
\hline 2 & Avignon & (Vaucluse) & Poirier & 1990 \\
\hline 3 & Avignon & (Vaucluse) & Cognassier & 1991 \\
\hline 4 & Avignon & (Vaucluse) & Pommier & 1991 \\
\hline 5 & Manosque & (Basses-Alpes) & Pommier & 1989 \\
\hline 6 & Manosque & (Basses-Alpes) & Noyer & 1989 \\
\hline 7 & Valence & (Drôme) & Pommier & 1989 \\
\hline 8 & Valence & (Drôme) & Noyer & 1989 \\
\hline 9. & Nyon & (Suisse) & Pommier & 1990 \\
\hline 10 & Saillon & (Suisse) & Abricotier & 1992 \\
\hline 11 & Saint-Marcellin & (Isère) & Poirier & 1991 \\
\hline 12 & Saint-Marcellin & (Isère) & Noyer & 1991 \\
\hline 13 & Saint-Colomban & (Savoie) & Pommier & 1991 \\
\hline 14 & Les Avanchers & (Savoie) & Pommier & 1992 \\
\hline 15 & Rennes & (Ille-et-Vilaine) & Pommier & 1990 \\
\hline 16 & Chambéry & (Savoie) & Pommier & 1990 \\
\hline 17 & Lille & (Nord) & Pommier & 1990 \\
\hline 18 & Montauban & (Tarn-et-Garonne) & Pommier & 1990 \\
\hline 19 & Strasbourg & (Bas-Rhin) & Pommier & 1990 \\
\hline
\end{tabular}

Distances entre plantes hôtes échantillonnées dans une même localité : plantes contiguës à Avignon et Saint-Marcellin, distantes de $0,5 \mathrm{~km}$ à Manosque, de $10 \mathrm{~km}$ à Valence. Les localités de Nyon et Saillon sont séparées par $200 \mathrm{~km}$. 
par ailleurs (Buès et Toubon, 1992)). Les échantillons 13 et 14 (Saint-Colomban et Les Avanchers), ont été prélevés dans des vallées alpines entre 1400 et $1600 \mathrm{~m}$ d'altitude.

\section{Élevages}

Afin de comparer au printemps les dates d'émergence des adultes issus de larves diapausantes récoltées dans différentes localités, les lots sont disposés, dès leur prélèvement à l'automne (septembre-octobre), sous abri insectarium à Avignon où ils sont soumis à des conditions voisines des conditions naturelles. Les localités et les dates de récolte sont indiquées dans les tableaux résumant les résultats. Les émergences ont été regroupées par classes de $5 \mathrm{j}$.

Pour comparer les pourcentages de diapause entre échantillons de localités différentes, les adultes éclos dans l'abri insectarium sont accouplés et alimentés avec de l'eau sucrée à $20 \%$. Malgré différentes techniques de conditionnement, les taux de femelles fertiles demeurent très faibles (voisins de $10 \%$ ). Nous n'avons donc pas toujours disposé d'un nombre de descendants suffisants pour tous les échantillons et conditions expérimentales.

\section{Caractères écophysiologiques}

Pour l'étude du seuil photopériodique d'induction de la diapause, la totalité des larves obtenues de chaque localité est répartie dans 3 conditions : LO (L : phase lumineuse, $\mathrm{O}$ : phase obscure) 15:9, 15,5:8,5, 16:8. L'élevage individuel a été réalisé à $25^{\circ} \mathrm{C}$ sur alimentation artificielle (Poitout et Buès, 1974). Les pourcentages de larves en diapause sont déterminés à partir du nombre de larves diapausantes observé 50 j après la mise en élevage.

Pour l'étude du seuil thermique de réactivation, $60 \mathrm{j}$ après la mise en élevage à $25^{\circ} \mathrm{C}$, LO $15: 9$, les lots d'insectes en diapause sont transférés à $2^{\circ} \mathrm{C}, \mathrm{LO}$ 12:12, pendant $100 \mathrm{j}$. Par la suite, chaque lot d'une localité donnée a été divisé en 3 et exposé à 18,21 et $25^{\circ} \mathrm{C}$, LO $16: 8$. Le pourcentage d'émergence est calculé $90 \mathrm{j}$ après l'exposition des lots aux 3 conditions. Les courbes d'émergences s'éloignant sensiblement de la normale pour certaines localités avec un faible effectif, les comparaisons ont été réalisées à l'aide d'un test non paramétrique (Kruskal-Wallis) (Scherrer, 1984).

\section{Analyse enzymatique}

Les analyses ont été effectuées sur les adultes, sur gel d'amidon pour 11 des 12 loci étudiés: Phosphoglucomutase (Pgm), Mannose-phosphate-isomérase (Mpi), Aspartate-amino-transférase (Aat+, Aat-), Isocitrate-deshydrogénase (ldh), Adénylate- kinase ( $A k)$, Hexokinase ( $H k$ ), enzyme malique $(M e)$, Malate-deshydrogénase ( $M d h), \alpha$-glycérophosphatedeshydrogénase ( $\alpha G p d$ ), Lactate-déshydrogénase $(L d h)$. Les procédures sont identiques à celles décrites précédemment (Buès et Toubon, 1992). Le zymogramme des estérases (Est) est réalisé sur gel d'acrylamide en plaque à $10 \%(\mathrm{~T} 24,6 \%, \mathrm{C} 2,4 \%)$ et révélé avec du $\alpha$ Naphthyl acétate. Un locus avec 8 allèles est nettement analysable, il est inhibé par un organophosphate (Mévinphos à $1 \%$ ) et non inhibé par un carbamate (Esérine à 1\%o).

Les fréquences alléliques aux loci polymorphes ont été comparées par le test G (Sokal et Rohlf, 1981). Pour cette analyse, les allèles rares sont regroupés avec l'allèle de mobilité la plus proche. Le test de conformité des structures génotypiques avec celles données par la loi de Hardy Weinberg, les distances génétiques (Nei, 1978) et l'algorithme UPGMA, sont calculés à l'aide de Biosys 1 (Swofford et Selander, 1981). Les statistiques $F$, leur écart type (méthode du Jackknife), et leur intervalle de confiance au seuil $5 \%$ (méthode du Bootstrap) ont été calculés à l'aide du programme Diploid (Weir, 1990). Dans ce modèle, la population totale est subdivisée en sous-populations, considérées comme partiellement isolées. L'éloignement génétique entre les sous-populations a été représenté à l'aide d'une analyse factorielle des correspondances.

Les flux géniques entre sous-populations, dans un modèle fragmenté en îlots, peuvent être estimés à l'aide du paramètre $N m$, où $(N)$ représente l'effectif de la population et $(m)$ le taux d'immigrants. $\mathrm{Nm}$ a été calculé d'après l'équation $\ln (p)=a \ln (\mathrm{Nm})+b$ (Slatkin, $1985)$, où ( $p$ ) est la fréquence moyenne des allèles privés (présents dans une seule des sous-populations), a et $b$ des coefficients. Slatkin a montré que pour $(p)$ compris entre 0,01 et 1 , les valeurs $a=0,504$ et $b=$ $-2,44$ donnaient une estimation satisfaisante de $\mathrm{Nm}$. Cet indice est corrigé en fonction de l'effectif moyen (n) analysé dans chaque sous-populations $\left(\mathrm{Nm}^{\prime}=\right.$ $25 \mathrm{Nm} / \mathrm{n}$ ). Une autre estimation des flux géniques a été effectuée suivant la méthode de Wright (1951), basée sur la relation inverse entre $\mathrm{Nm}$ et $F s t$, où $\mathrm{Nm}=$ $\{(1 / F s t)-1\} / 4$.

\section{RÉSULTATS}

\section{Élevages sous abri insectarium}

L'échelonnement des dates d'émergence au printemps, ainsi que la date à laquelle $50 \%$ des émergences ont eu lieu, sont indiqués dans le tableau II. Elles varient en fonction des années, indépendamment de l'origine géographique ou de la plante hôte, en liaison avec les températures moyennes. En revanche, pour une année donnée, les dates d'émergences sont sensiblement identiques, aussi bien entre origines géo- 
Tableau II. Dates d'émergence au printemps, de 50\% des adultes issus de larves diapausantes de différentes origines, récoltées à l'automne sur pommier, noyer, poirier, abricotier et cognassier et placées sous abri insectarium à Avignon.

\section{Origine}

1989

Manosque

Valence

Valence

1990

Rennes

Chambéry

Nyon

Lille

Strasbourg

Montauban

Manosque

Avignon

1991

Avignon

Avignon

Saint-Colomban

Valence

Valence

Saint-Marcellin

1992

Avignon
Les Avanchers
Avignon
Saillon

Plante hôte

Pommier

Pommier

Noyer

Pommier

Pommier

Pommier

Pommier

Pommier

Pommier

Pommier

Pommier

Pommier

Cognassier

Pommier

Pommier

Noyer

Noyer

Pommier

Pommier

Poirier

Abricotier
$N$

209

158

367

1996

261

316

256

145

366

136

562

1294

180

37

245

998

461

134

33

31

49
Date d'émergence a

13/06 (6/5-30/6)

$13 / 06(21 / 5-30 / 6)$

$13 / 06(16 / 5-5 / 7)$

$23 / 05(6 / 5-30 / 6)$

23/05 (6/5-20/6)

$23 / 05(6 / 5-20 / 6)$

$23 / 05(6 / 5-15 / 6)$

$28 / 05(6 / 5-25 / 6)$

$28 / 05(1 / 5-30 / 6)$

$10 / 05(1 / 5-10 / 6)$

$18 / 05(6 / 5-5 / 7)$

$8 / 06(6 / 5-10 / 7)$

$3 / 06(11 / 5-10 / 7)$

$8 / 06(26 / 5-20 / 6)$

$13 / 06(11 / 5-10 / 7)$

$13 / 06(6 / 5-10 / 7)$

$18 / 06(21 / 5-10 / 7)$

$13 / 05(1 / 5-15 / 6)$

$13 / 05(1 / 5-5 / 6)$

$13 / 05(6 / 5-31 / 5)$

$8 / 05(1 / 5-5 / 6)$

a Entre parenthèses : dates de la première et de la dernière émergence.

graphiques qu'entre cultures hôtes, excepté en 1991 entre Avignon pommier et Saint-Marcellin noyer. Les émergences de l'échantillon de SaintColomban pommier sont relativement précoces (7/06), mais ne concernent qu'un faible nombre d'individus par rapport aux autres lots. L'échantillon de la population des Avanchers, prélevé en 1992 dans des conditions environnementales voisines de celles de Saint-Colomban, n'est pas différent des autres.

\section{Élevages en conditions constantes}

Les pourcentages de larves en diapause, observés sous LO 15:9 et 15,5:8,5 (tableau III), varient très faiblement quels que soient la localité ou l'hôte : 98,8 à $100 \%$ sous LO $15: 9$ et 83,8 à $100 \%$ sous LO 15,5:8,5. Une exception concerne la population de Montauban sous LO 15,5:8,5 $(43,3 \%)$ mais cette différence n'a pas pu être vérifiée dans les autres conditions. Sous LO 16:8, les écarts sont plus importants : de $24,4 \%$ pour la population de Manosque à $78,7 \%$ pour celle de Rennes. Pour la population d'Avignon, la différence entre pommier et poirier n'est pas significative statistiquement (test basé sur l'écart réduit). En revanche, les différences entre les échantillons récoltés, soit sur noyer à Valence et SaintMarcellin (localités géographiquement peu éloignées), soit sur pommier à Rennes et Chambéry, sont significatives. Des élevages, conduits de la même façon sous LO 17:7, permettent de confirmer un taux de diapause plus faible dans la population de Manosque (4,1\%) que dans celles d'Avignon, Chambéry et Rennes (14 à 15\%).

Les durées moyennes de réactivation des larves diapausantes à 18,21 et $25^{\circ} \mathrm{C}$, LO 16:8, 
Tableau III. Pourcentages de diapause induite à $25^{\circ} \mathrm{C}$ (LO 15:9, 15,5:8,5 et 16:8) chez des lots d'insectes d'origines différentes.

Origine

$15: 9$

Valence
Saint-Marcellin
Avignon
Avignon
Manosque
Rennes
Chambéry
Montauban
Nyon
Lille

* Entre parenthèses, le nombre d'individus émergés ou à l'état larvaire, 50 j après la mise en élevage.

(noyer)
(noyer)
(poirier)
(pommier)
(pommier)
(pommier)
(pommier)
(pommier)
(pommier)
(pommier)

100
100
100
99,5
98,8
100
100
$/$
1
100

$(332)^{\star}$

(76)

(81)

$(380)$

(84)

(55)

(65)

(13)

98,0
94,1
89,5
89,6
88,4
96,6
83,8
43,3
100
100

Pourcentages de diapause sous $L O$

$15,5: 8,5$

$16: 8$

$\begin{array}{cc} \\ 01) & 48,7 \\ 02) & 29,3 \\ (86) & 34,7 \\ 624) & 30,9 \\ 12) & 24,4 \\ 58) & 78,7 \\ 68) & 62,5 \\ 30) & / \\ 73) & / \\ 48) & 1\end{array}$

(119)

(80) sont indiquées dans le tableau IV. Les résultats du test de Kruskal-Wallis, réalisé pour chacune des conditions, montre une hétérogénéité significative entre les origines et les plantes hôtes (à $25^{\circ} \mathrm{C}, \mathrm{H}=17,876$; à $21^{\circ} \mathrm{C}, \mathrm{H}=55,427$; à $18^{\circ} \mathrm{C}$, $H=41,042$; ddl =7). Toutefois, les tests de comparaisons multiples, réalisés à partir des sommes de rangs pour les 3 conditions, ne permettent pas de différencier les moyennes au seuil $5 \%$ et l'on ne peut que conclure à une hétérogénéité globale entre les localités et les plantes hôtes.

\section{Analyse enzymatique}

Le tableau $\mathrm{V}$ donne les fréquences alléliques de 12 échantillons de populations. Sept loci sur 12 sont polymorphes (fréquence du $2^{e}$ allèle $>1 \%$ ) : Pgm, Mpi, Idh, Aat t, Aat-, Ak et Est. L'hétérozygotie moyenne observée varie entre 0,178 (Avignon, poirier) et 0,292 (Avignon, pommier). Sur 84 analyses de conformité des fréquences génotypiques observées avec celles de Hardy Weinberg, 3 fournissent des différences significatives au seuil $1 \%$ au locus Aatt, une au seuil $1 \%$ au locus $A k$ et deux au seuil $5 \%$ aux loci Pgm et Est. Les valeurs des Fis et des Fit, qui mesurent respectivement le déséquilibre des fréquences génotypiques dans les sous-populations et dans la population totale, sont indiquées pour chaque locus dans le tableau VI. Les Fis confirment le déficit d'hétérozygotes (génotype bc) au locus Aatt, dans les sous-populations de Manosque sur pommier et noyer. II est remar- quable que l'allèle c des loci Aat+ et Aat-, ne soit présent que dans les populations de Manosque et Valence, indépendamment de la plante hôte.

Le tableau VI indique également pour chaque locus les valeurs de Fst, qui mesure le degré de différenciation génétique entre les sous-populations. Fst varie de 0,0005 à 0,0592 selon les locus avec une moyenne de 0,0281 . Il présente un écart type de 0,0085 et un intervalle de confiance à $95 \%$ de 0,0177 à 0,049 9. Le Fst calculé entre les échantillons de 4 sous-populations géographiquement éloignées (Avignon, Valence, Manosque et Nyon), récoltés sur pommier exclusivement, est de 0,029 4 (avec un écart type important, 0,022 7, dû au seul locus $A k$ ). Le même calcul effectué entre 4 échantillons prélevés à Avignon (pommier, 1991 ; poirier, 1990 et 1991 ; cognassier, 1991) indique un Fst de 0,0385 (écart type : 0,014 1). La différence entre les 2 Fst est significative au seuil 1\% (test $U$ de Mann-Whitney-Wilcoxon, réalisé sur les 7 loci polymorphes). Les valeurs des distances génétiques non biaisées (Nei, 1978), de 0 à 0,018 , portées sur le phénogramme (fig 1), indiquent une faible divergence entre populations. Seule la population de Saillon, sur abricotier, se différencie faiblement des autres. Les moyennes des distances génétiques calculées entre d'une part, les 4 échantillons récoltés sur pommier dans des localités différentes $(0,0047)$, et d'autre part, les 4 échantillons prélevés à Avignon sur différentes plantes hôtes $(0,0082)$, confirment les résultats obtenus avec le Fst. L'AFC (fig 2) montre que les échantillons de Manosque et 
Tableau IV. Durées moyennes de réactivation en jours ( \pm écart type) à 18,21 et $25^{\circ} \mathrm{C}$ (LO 16:8), de lots de larves diapausantes d'origines géographiques différentes, obtenus à $25^{\circ} \mathrm{C}$ (LO 15:9) et préalablement exposés à $2^{\circ} \mathrm{C}$ (LO 12:12) pendant $100 \mathrm{j}$.

Origine

$$
18^{\circ}
$$

Avignon (pommier)

$$
47,3 \pm 13,1
$$

$$
\text { Avignon (poirier) }
$$

$54,5 \pm 12,5$

Valence (noyer)

$46,7 \pm 11,8$

Saint-Marcellin (noyer)

$46,2 \pm 8,8$

Rennes (pommier)

$54,3 \pm 14,7$

Chambéry (pommier)

$52,1 \pm 11,4$

$65,9 \pm 13,7$

Manosque (pommier)

$48,5 \pm 8,8$

(24)

\section{Durée de développement à}

$21^{\circ}$

$36,4 \pm 6,7$

(76)

$43,1 \pm 11,8$

(47)

$36,0 \pm 9,5$

(78)

$32,7 \pm 6,9$

(26)

$43,4 \pm 15,6$

(15)

$46,6 \pm 17,9$

(26)

$45,1 \pm 15,2$

(17)

$35,3 \pm 8,8$

(21)
25

$31,6 \pm 7,0$

$(74)^{\star}$

$30,9 \pm 5,9$

(38)

$29,7 \pm 6,9$

$28,8 \pm 6,9$

$34,2 \pm 13,7$

$36,1 \pm 14,1$

$36,9 \pm 12,4$

$33,9 \pm 13,4$

Entre parenthèses le nombre d'adultes éclos plus le nombre de larves encore en diapause, $90 \mathrm{j}$ après l'exposition à $18,21,25^{\circ} \mathrm{C}$ (LO 16:8). Les résultats du test de Kruskal-Wallis sont indiqués dans le texte.

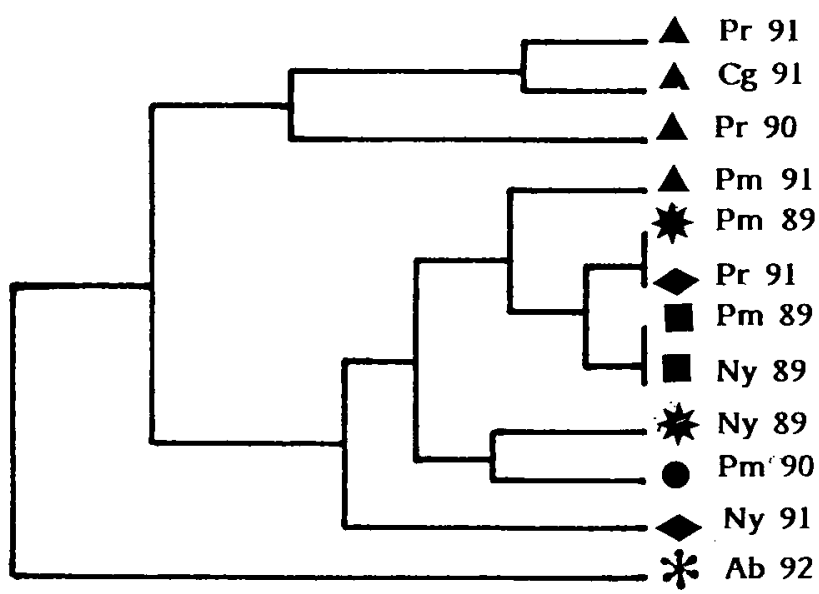

0,010

Distance génétique
Fig 1. Phénogramme UPGMA regroupant 12 échantillons, établi à partir des distances génétiques de Nei calculées sur 7 loci polymorphes ( $\boldsymbol{\Delta}$ : Avignon, * : Manosque, $\mathbf{\square}$ : Valence, $\bullet$ : Nyon, * : Saillon, : SaintMarcellin ; $\mathrm{Pr}$ : poirier, $\mathrm{Pm}$ : pommier, $\mathrm{Cg}$ : cognassier, $\mathrm{Ny}$ : noyer, Ab : abricotier, l'indication de la localité et de la plante hôte est suivie de l'année de prélèvement). 
Tableau V. Fréquences alléliques à 7 loci examinés chez les échantillons 1 à 12 (cf tableau I). Nombre d'hétérozygotes observés (obs) et attendus (att), signification au seuil de ${ }^{*} 5 \%,{ }^{\star *} 1 \%,{ }^{* \star *} 1 \%$ (test de probabilités exactes), hétérozygotie moyenne observée et attendue.

Locus

$N^{\circ}$ de l'échantillon (cf tableau l)

$\begin{array}{llllllllllll}1 & 2 & 3 & 4 & 5 & 6 & 7 & 8 & 9 & 10 & 11 & 12\end{array}$

\begin{tabular}{|c|c|c|c|c|c|c|c|c|c|c|c|c|}
\hline \multicolumn{13}{|l|}{ Pgm } \\
\hline$n$ & 48 & 83 & 48 & 48 & 308 & 119 & 102 & 144 & 95 & 51 & 48 & 24 \\
\hline a & 0,115 & 0,072 & 0,177 & 0,094 & 0,149 & 0,071 & 0,108 & 0,097 & 0,074 & 0,049 & 0,135 & 0,165 \\
\hline$b$ & 0,635 & 0,428 & 0,531 & 0,531 & 0,432 & 0,647 & 0,500 & 0,514 & 0,605 & 0,725 & 0,428 & 0,438 \\
\hline c & 0,240 & 0,446 & 0,177 & 0,344 & 0,330 & 0,252 & 0,353 & 0,351 & 0,247 & 0,157 & 0,375 & 0,333 \\
\hline$d$ & 0,010 & 0,054 & 0,115 & 0,031 & 0,089 & 0,030 & 0,039 & 0,038 & 0,074 & 0,069 & 0,032 & 0,064 \\
\hline obs & 31 & 42 & 33 & 32 & 200 & 56 & 64 & 82 & 50 & 24 & 28 & 19 \\
\hline att & 25 & $51^{*}$ & 31 & 29 & 208 & 61 & 63 & 87 & 54 & 23 & 31 & 16 \\
\hline \multicolumn{13}{|l|}{ Ak } \\
\hline$n$ & 48 & 83 & 48 & 48 & 260 & 144 & 150 & 144 & 96 & 51 & 48 & 23 \\
\hline a & 0,010 & 0,012 & 0,000 & 0,010 & 0,080 & 0,003 & 0,020 & 0,014 & 0,016 & 0,059 & 0,063 & 0,000 \\
\hline b & 0,886 & 0,880 & 0,906 & 0,636 & 0,702 & 0,695 & 0,727 & 0,757 & 0,828 & 0,804 & 0,697 & 0,826 \\
\hline c & 0,104 & 0,108 & 0,094 & 0,354 & 0,212 & 0,302 & 0,243 & 0,215 & 0,156 & 0,137 & 0,240 & 0,174 \\
\hline d & 0,000 & 0,000 & 0,000 & 0,000 & 0,006 & 0,000 & 0,010 & 0,014 & 0,000 & 0,000 & 0,000 & 0,000 \\
\hline obs & 11 & 20 & 9 & 30 & 109 & 58 & 65 & 55 & 21 & 16 & 25 & 6 \\
\hline att & 10 & 18 & 8 & 23 & 119 & 62 & 62 & 55 & $28^{\star \star}$ & 17 & 22 & 7 \\
\hline \multicolumn{13}{|l|}{ Est } \\
\hline$n$ & 72 & 67 & 48 & 70 & 107 & 148 & 128 & 149 & 71 & 51 & 61 & 33 \\
\hline a & 0,000 & 0,000 & 0,000 & 0,000 & 0,005 & 0,003 & 0,008 & 0,013 & 0,000 & 0,010 & 0,000 & 0,000 \\
\hline b & 0,000 & 0,000 & 0,042 & 0,000 & 0,033 & 0,051 & 0,039 & 0,020 & 0,014 & 0,020 & 0,016 & 0,015 \\
\hline c & 0,666 & 0,723 & 0,760 & 0,572 & 0,569 & 0,531 & 0,538 & 0,518 & 0,522 & 0,451 & 0,607 & 0,546 \\
\hline d & 0,035 & 0,022 & 0,031 & 0,164 & 0,103 & 0,091 & 0,051 & 0,067 & 0,070 & 0,176 & 0,098 & 0,258 \\
\hline e & 0,229 & 0,231 & 0,125 & 0,207 & 0,229 & 0,297 & 0,313 & 0,332 & 0,331 & 0,186 & 0,246 & 0,136 \\
\hline f & 0,056 & 0,22 & 0,042 & 0,043 & 0,014 & 0,027 & 0,051 & 0,040 & 0,063 & 0,147 & 0,025 & 0,045 \\
\hline $\mathrm{g}$ & 0,014 & 0,000 & 0,000 & 0,014 & 0,047 & 0,000 & 0,000 & 0,010 & 0,000 & 0,010 & 0,008 & 0,000 \\
\hline obs & 37 & 27 & 21 & 41 & 62 & 90 & 66 & 83 & 38 & 27 & 31 & 14 \\
\hline att & 36 & 28 & 19 & 42 & 65 & 92 & $78^{\star}$ & 92 & 44 & 36 & 35 & 21 \\
\hline \multicolumn{13}{|l|}{ Mpi } \\
\hline$n$ & 48 & 83 & 48 & 48 & 96 & 96 & 48 & 24 & 94 & 48 & 48 & 24 \\
\hline a & 0,063 & 0,030 & 0,042 & 0,052 & 0,000 & 0,000 & 0,011 & 0,021 & 0,000 & 0,010 & 0,021 & 0,083 \\
\hline b & 0,916 & 0,952 & 0,958 & 0,938 & 1,000 & 1,000 & 0,978 & 0,979 & 0,979 & 0,928 & 0,969 & 0,917 \\
\hline c & 0,021 & 0,018 & 0,000 & 0,010 & 0,000 & 0,000 & 0,011 & 0,000 & 0,021 & 0,031 & 0,010 & 0,000 \\
\hline d & 0,000 & 0,000 & 0,000 & 0,000 & 0,000 & 0,000 & 0,000 & 0,000 & 0,000 & 0,031 & 0,000 & 0,000 \\
\hline obs & 7 & 8 & 4 & 5 & I & 1 & 2 & 1 & 4 & 7 & 3 & 4 \\
\hline att & 8 & 8 & 4 & 6 & 1 & 1 & 2 & 1 & 4 & 7 & 3 & 4 \\
\hline \multicolumn{13}{|l|}{ Aat+ } \\
\hline $\mathrm{n}$ & 48 & 83 & 48 & 48 & 312 & 144 & 126 & 120 & 95 & 51 & 48 & 24 \\
\hline a & 0,010 & 0,000 & 0,000 & 0,000 & 0,008 & 0,017 & 0,008 & 0,000 & 0,005 & 0,000 & 0,000 & 0,000 \\
\hline b & 0,990 & 1,000 & 1,000 & 1,000 & 0,965 & 0,976 & 0,992 & 0,983 & 0,995 & 1,000 & 1,000 & 1,000 \\
\hline c & 0,000 & 0,000 & 0,000 & 0,000 & 0,027 & 0,007 & 0,000 & 0,017 & 0,000 & 0,000 & 0,000 & 0,000 \\
\hline obs & 1 & I & 1 & I & 8 & 3 & 2 & 0 & 1 & 1 & I & 1 \\
\hline att & 1 & 1 & 1 & 1 & $21^{\star \star \star}$ & $7^{\star \star \star}$ & 2 & $4^{\star \star \star *}$ & 1 & 1 & 1 & I \\
\hline \multicolumn{13}{|l|}{ Aat- } \\
\hline$n$ & 48 & 83 & 48 & 48 & 120 & 144 & 78 & 48 & 96 & 51 & 48 & 24 \\
\hline a & 0,000 & 0,000 & 0,000 & 0,000 & 0,000 & 0,000 & 0,000 & 0,010 & 0,000 & 0,000 & 0,000 & 0,000 \\
\hline b & 1,000 & 1,000 & 1,000 & 1,000 & 0,992 & 0,993 & 0,981 & 0,990 & 1,000 & 1,000 & 1,000 & 1,000 \\
\hline $\mathrm{C}$ & 0,000 & 0,000 & 0,000 & 0,000 & 0,008 & 0,007 & 0,019 & 0,000 & 0,000 & 0,000 & 0,000 & 0,000 \\
\hline obs & I & 1 & I & I & 2 & 2 & 3 & 1 & 1 & 1 & 1 & 1 \\
\hline att & 1 & 1 & I & 1 & 2 & 2 & 3 & 1 & 1 & 1 & 1 & I \\
\hline \multicolumn{13}{|l|}{ Idh } \\
\hline$n$ & 48 & 72 & 48 & 48 & 188 & 144 & 150 & 144 & 95 & 51 & 48 & 24 \\
\hline a & 0,000 & 0,000 & 0,000 & 0,000 & 0,003 & 0,000 & 0,000 & 0,000 & 0,000 & 0,000 & 0,000 & 0,000 \\
\hline b & 1,000 & 1,000 & 0,990 & 0,969 & 0,978 & 0,969 & 0,987 & 0,990 & 0,963 & 1,000 & 0,979 & 0,979 \\
\hline $\mathrm{c}$ & 0,000 & 0,000 & 0,010 & 0,031 & 0,019 & 0,031 & 0,013 & 0,010 & 0,037 & 0,000 & 0,021 & 0,021 \\
\hline obs & 1 & 1 & 1 & 3 & 6 & 9 & 4 & 3 & 7 & 1 & 2 & 1 \\
\hline att & 1 & 1 & 1 & 3 & 8 & 9 & 4 & 3 & 7 & 1 & 2 & 1 \\
\hline \multicolumn{13}{|c|}{ Hétérozygotie moyenne observée $(H o)$ et écart type $( \pm \sigma)$} \\
\hline Ho & 0,222 & 0,178 & 0,202 & 0,292 & 0,246 & 0,226 & 0,243 & 0,227 & 0,201 & 0,209 & 0,245 & 0,241 \\
\hline$\pm \sigma$ & 0,099 & 0,079 & 0,100 & 0,119 & 0,110 & 0,098 & 0,102 & 0,100 & 0,089 & 0,087 & 0,104 & 0,109 \\
\hline \multicolumn{13}{|c|}{ Hétérozygoptie moyenne attendue $(\mathrm{Ht})$ et écart type $( \pm \sigma)$} \\
\hline $\mathrm{Ht}$ & 0,203 & 0,193 & 0,190 & 0,265 & 0,267 & 0,241 & 0,251 & 0,246 & 0,228 & 0,234 & 0,252 & 0,257 \\
\hline$\pm \sigma$ & 0,087 & 0,092 & 0,094 & 0,106 & 0,114 & 0,102 & 0,107 & 0,106 & 0,101 & 0,105 & 0,109 & 0,110 \\
\hline
\end{tabular}


Tableau VI. Valeurs des statistiques $F$ pour chaque locus, calculées sur l'ensemble des prélèvements 1 à 12.

\begin{tabular}{lrrr} 
Locus & \multicolumn{1}{c}{ FIS } & FIT & FST \\
& & & \\
Pgm & 0,0258 & 0,0460 & 0,0207 \\
Ak & $-0,0168$ & 0,0434 & 0,0592 \\
Est & 0,0858 & 0,1088 & 0,0252 \\
Mpi & 0,2640 & 0,2747 & 0,0145 \\
Aat+ & 0,4671 & 0,4681 & 0,0019 \\
Aat- & 0,1378 & 0,1383 & 0,0005 \\
Idh & 0,0352 & 0,0385 & 0,0034 \\
MOY & 0,0526 & 0,0791 & 0,0281 \\
Écart type & 0,0292 & 0,0269 & 0,0085 \\
Intervalle de confiance $(95 \%)$ & & \\
$\quad+$ & 0,1316 & 0,1507 & 0,0177 \\
$\quad-$ & 0,0109 & 0,0481 & 0,0499
\end{tabular}

Valence sur pommier et noyer sont assez nettement isolés des autres. Ce résultat est dû à la présence d'allèles à des fréquences relativement élevées, notamment des allèles $\mathrm{c}$ des loci Aat+ et Aat-, a et d du locus Pgm. Notons que le $1^{\mathrm{er}}$ axe absorbe $42,7 \%$ et le $2^{\mathrm{e}} 19,1 \%$ de l'inertie totale.

Dans le tableau VII sont présentés les résultats des tests $G$ comparant, à partir des fréquences alléliques aux 6 loci les plus polymorphes (Pgm, Est, Ak, Aat+, Idh, Mpi), les sous-populations récoltées sur des plantes hôtes différentes. Dans la comparaison pommier/noyer chez la sous-population de Manosque, on obser- ve des différences significatives pour 4 loci ( $P g m$, $A k$, Est, Aat+). Des différences hautement significatives sont également observées dans la localité d'Avignon entre échantillons prélevés sur pommier, poirier et cognassier, malgré la position contiguë des cultures hôtes dans la parcelle. On note également dans la même localité et sur la même plante (poirier), une différence significative entre années au locus Pgm. En revanche, on n'observe qu'une faible différence entre les prélèvements effectués sur pommier et abricotier en Suisse, malgré notamment la présence au locus Mpi d'un allèle supplémentaire $d$.

Le calcul des flux géniques, entre sous-populations issues de plantes hôtes différentes, dans une même localité au cours d'une même année, suivant la méthode des allèles privés (tableau VII), montre que des échanges d'individus ont lieu dans les localités de Valence entre pommier et noyer $\left(\mathrm{Nm}^{\prime}=13,24\right)$ et d'Avignon entre pommier et poirier $\left(\mathrm{Nm}^{\prime}=9,15\right)$ et entre poirier et cognassier $\left(\mathrm{Nm}^{\prime}=11,62\right)$. L'estimation globale selon la méthode de Wright (1951), pour un Fst moyen de 0,029, fait apparaître un indice $\mathrm{Nm}$ variant en fonction de l'écart type entre 6 et 12. Les valeurs de $N m$, calculées à partir des Fst obtenus entre d'une part les échantillons récoltés sur pommier dans 4 localités éloignées $(\mathrm{Nm}=8)$, et d'autre part entre les 4 échantillons prélevés sur diverses plantes hôtes à Avignon $(N m=6)$, sont très proches. D'après Slatkin (1987), ce nombre représente un flux génique suffisamment important pour homogénéiser significativement les fréquences alléliques.

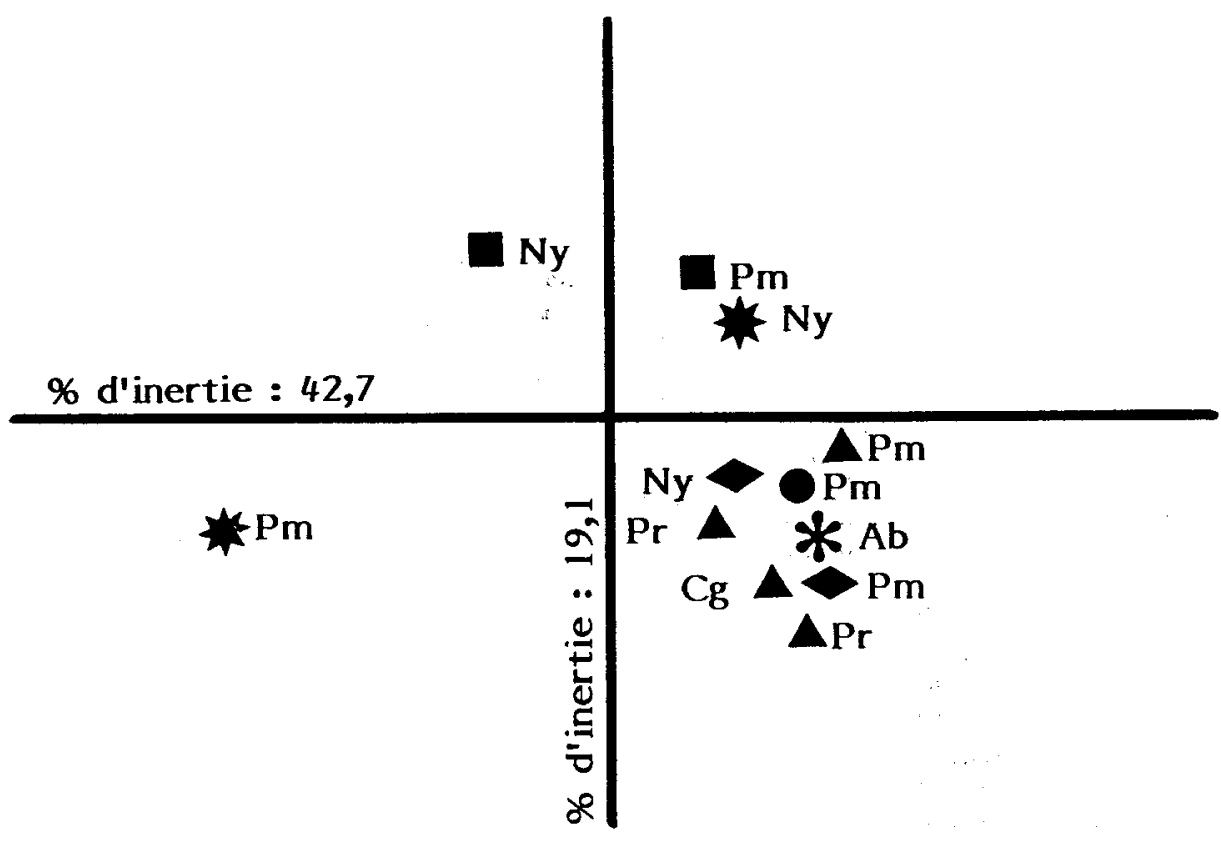

Fig 2. Analyse factorielle des correspondances entre les fréquences de 25 allèles ( 7 loci polymorphes) et 12 échantillons, prélevés sur des hôtes différents ( $\boldsymbol{\Delta}$ : Avignon, * : Manosque, $\mathbf{\square}$ : Valence, - Nyon, *: Saillon, Saint-Marcellin ; $\mathrm{Pr}$ : poirier $\mathrm{Pm}$ : pommier, $\mathrm{Cg}$ : cognas sier, Ny : noyer, Ab : abricotier, pour l'année de prélèvement se reporter au tableau I). 
Tableau VII. Comparaison des fréquences alléliques aux 6 loci les plus polymorphes, entre échantillons récoltés sur divers hôtes et flux génique (Slatkin, 1985), $N m^{\prime}$ n'a pas été calculé dans les 2 cas d'observations réalisées au cours d'années différentes.

Localité

Hôtes

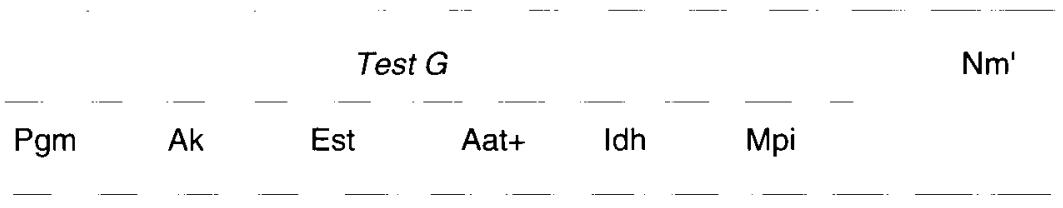

Manosque (1989)

Valence (1989)

Suisse (1990/1992)

Saint-Marcellin (1991)

Avignon (1990-1991)

Avignon (1991)

Avignon (1991)

Avignon (1991)

$\begin{array}{lcc}\text { Pom/Noy } & 38,1^{\star \star \star} & 34,8^{\star \star \star} \\ \text { Pom/Noy } & 0,2 & 0,9 \\ \text { Pom/Abr } & 4,8 & 4,0 \\ \text { Moy/Poi } & 1,1 & 6,0 \\ \text { Poi/Poi } & 16,6^{\star \star \star} & 0,1 \\ \text { Pom/Poi } & 3,2 & 17,7^{\star \star \star} \\ \text { Pom/Cog } & 14,5^{\star \star} & 21,2^{\star \star \star} \\ \text { Poi/Cog } & 12,8^{\star \star} & 1,4\end{array}$

${ }^{\star}$ Significatif au seuil de ${ }^{*} 5 \%,{ }^{* *} 1 \%,{ }^{* *} 1 \%$.

$\begin{array}{cc}21,8^{\star \star \star} & 6,2^{\star} \\ 13,3^{\star} & 8,4^{\star} \\ 13,5^{\star} & 3,4 \\ 10,9 & / \\ 4,4 & 1,9 \\ 12,3^{\star} & 1,4 \\ 25,4^{* \star \star} & 1 \\ 10,9 & 1,4\end{array}$

5,8
0,1
$6,1^{*}$
$/$
0,01
4,2
1,1
1,4

\section{5,8}

/

I

1,67

13,24

I

3,63

I

9,15

0,11

11,62

\section{DISCUSSION ET CONCLUSIONS}

Selon l'origine des échantillons, des différences apparaissent au niveau soit des taux d'induction de la diapause $\left(25^{\circ} \mathrm{C}\right.$, LO 16:8), soit des durées de réactivation post-diapause $\left(18\right.$ et $21^{\circ} \mathrm{C}$, LO $16: 8$ ), indépendamment de la plante hôte. En revanche, pour une année donnée, aucune différence réellement significative n'est observée entre les dates moyennes d'émergence, au printemps sous insectarium. II est probable que, dans les conditions naturelles, l'accroissement rapide des températures au printemps se traduise par un regroupement des émergences, comme celà est observé à $25^{\circ} \mathrm{C}$. Bien que nous ne disposions, dans une même zone géographique, que de très peu de comparaisons, aucune différence probante n'est observée entre les seuils photopériodiques d'induction de la diapause ou les vitesses de réactivation post-diapause, en fonction de la plante hôte. L'origine géographique semble seule intervenir. Ce résultat coïncide avec les observations de Sel'Deshova (1967) ou de Rield et Croft (1978). Ces derniers indiquent qu'à une augmentation de $10^{\circ}$ de latitude correspond un accroissement de 1,25 h des seuils photopériodiques, ceux-ci variant également, dans une même aire, en fonction des conditions subies avant la diapause. Dans les limites géographiques de cette étude, il ne semble pas exister de réels gradients latitudinaux ni des seuils photopériodiques d'induction ni des seuils thermiques de réactivation, mais plutôt des différences régionales. Par ailleurs, Phillips et
Barnes (1975) indiquent que l'émergence des individus sur prunier s'effectue plus tôt que celle sur noyer et pommier. El Gamil et al (1978) et Steinberg et al (1992) trouvent également une influence de l'alimentation, respectivement sur la vitesse de développement et l'induction de la diapause. En revanche, Pitcairn et al (1992) n'observent des différences significatives qu'entre localités mais pas entre cultures hôtes. Ce dernier résultat corrobore nos propres observations. Cependant, une étude conduite à $25^{\circ} \mathrm{C}, \mathrm{LO}$ $15,5: 8,5$, sur 15 descendances de couples isolés de la population d'Avignon (individus issus de la génération hivernante), montre une grande variabilité entre descendances (taux de diapause des lignées regroupées : $85,9 \%$ avec un maximum et un minimum respectivement de 91,9 et $22,9 \%$ ). Cette variabilité peut expliquer les conclusions contradictoires auxquelles parviennent les auteurs.

Les résultats de l'analyse enzymatique font apparaître une bonne concordance des fréquences génotypiques avec celles de la loi de Hardy Weinberg, excepté pour le locus Aat+ dans les localités de Manosque sur noyer et pommier et Valence sur noyer. Dans ces échantillons, on observe un déficit d'hétérozygotes et la présence d'un allèle $c$. Ce déséquilibre se retrouve dans le calcul des paramètres Fis et Fit, fortement positifs. La présence d'un allèle supplémentaire dans ces 2 localités s'observe aussi pour le locus Aat-, indépendamment de la culture. Ces résultats sont également observables au niveau de I'AFC. Dans une précédente étude 
(Buès et Toubon, 1992), l'allèle $c$ des loci Aat à migration cathodique ou anodique était également observé dans d'autres localités (Orléans, Rennes, Montauban...) et interprété comme indicateur d'une variabilité géographique. II en est de même pour l'allèle $\mathrm{d}$ du locus Mpi, observé dans la sous-population Suisse sur abricotier, qui avait précédemment été noté dans les populations de Rennes et de Saint-Colomban sur pommier (Buès et Toubon, 1992). Des différences significatives de fréquences alléliques sont observées dans la localité de Manosque, entre pommier et noyer au niveau des loci Pgm, AK et Est. Dans cette localité, les échantillons ont été récoltés sur quelques noyers isolés et sur des pommiers distants d'environ $500 \mathrm{~m}$. Le nombre d'individus analysés est important et la lecture des zymogrammes sans ambiguité. II est alors probable que les différences constatées proviennent d'un "effet fondateur» sur les noyers (individus issus de la population sur pommier). Le relativement faible flux génique observé tendrait à confirmer cette hypothèse et à expliquer l'absence sur noyer, notamment de l'allèle $d$ du locus $A k$ et de l'allèle $\mathrm{g}$ du locus Est. Toutefois, il est improbable que l'isolement de cette fraction de la population se maintienne au-delà de la période estivale. La coïncidence, au printemps, des émergences et le décalage des stades phénologiques sensibles des plantes hôtes conduisent à un brassage au moins annuel des sous-populations.

Des différences significatives sont également observées, à l'aide du test $\mathrm{G}$, entre échantillons récoltés à Avignon en 1991 sur pommier/poirier et pommier/cognassier. La variété de poire échantillonnée (Guyot) est récoltée dès juillet, ce qui limite les attaques à la $1^{\text {re }}$ génération et oblige la $2^{e}$ génération à s'installer essentiellement sur pommier et cognassier (ce dernier n'est que faiblement attaqué au cours de la $1^{\text {re }}$ génération). Compte tenu de la proximité des parcelles des 3 cultures hôtes et du fait que la diapause hivernale provoque un regroupement des émergences au printemps, on peut considérer, comme à Manosque sur pommier et noyer, que c'est la même population qui est à l'origine des attaques. Le pommier étant l'hôte principal le plus répandu dans la zone géographique considérée, on peut supposer que c'est une fraction de la même population, plus ou moins importante selon les années, qui effectue une génération et rarement 2 consécutives, sur poirier ou cognassier. Phillips et Barnes (1975) ont montré qu'il existait des préférences d'oviposition des adultes, d'ordre génétique, en fonction du conditionnement préimaginal. On pourrait supposer que ce comportement conduise comme chez Rhagoletis pomonella Walsh (Feder et al, 1988) à la formation de races, mais ce n'est pas le cas ici. Les distances génétiques entre échantillons, calculées à partir de 7 loci polymorphes varient seulement de 0 à 0,025 avec une moyenne de 0,007 (écart type $=0,005$ ). Les mêmes calculs effectués à partir des mêmes 7 loci sur 12 populations d'origines géographiques différentes, récoltées uniquement sur pommier (Buès et Toubon, 1992) ont donné une moyenne de 0,002 (écart type $=0,002$ ). Cette étude a conduit à un Fst de 0,015 0 (écart type $=0,010$ ) contre 0,0281 (écart type $=0,0085$ ) d'après les résultats présentés ci-dessus. Pashley (1985) indique un Fst de 0,066, calculé à partir de 4 loci, entre populations de cette même espèce d'Afrique, d'Europe, des États-Unis et d'Australie.

La différenciation génétique, révélée par les marqueurs utilisés dans cette étude, est en moyenne légèrement plus importante lorsque les échantillons sont prélevés sur plusieurs plantes hôtes plutôt que sur une seule, la plus répandue (pommier). Les différences génétiques observées ne permettent cependant pas de discriminer les échantillons en fonction de l'hôte d'origine. L'absence de différences écophysiologiques entre échantillons provenant d'hôtes différents et l'existence d'un flux génique important renforcent l'hypothèse de populations essentiellement inféodées au pommier et de migrations d'une partie des individus vers des plantes hôtes plus occasionnelles, en fonction de coïncidences spatiotemporelles. En revanche, les différences écophysiologiques et enzymatiques constatées entre localités, semblent indiquer un processus d'adaptation aux conditions locales. Le flux génique est plus réduit entre localités éloignées et rend possible une différenciation géographique. Cependant, les échanges entre sous-populations sont encore suffisamment importants pour que la résistance au Diflubenzuron, récemment apparue dans la population de $C$ pomonella dans le sudest de la France (Sauphanor et al, 1994), puisse s'étendre et constituer un risque élevé de généralisation de cette résistance.

\section{REMERCIEMENTS}

Les auteurs remercient les lecteurs anonymes pour leurs remarques, ainsi que $\mathrm{J}$ Freuler, $\mathrm{M}$ Blanc et les personnes du service de la Protection des végétaux qui ont bien voulu leur adresser des échantillons. Ils remercient également $L$ Boudinhon pour sa participation aux élevages. 


\section{RÉFÉRENCES}

Bovey $P$ (1966) Le carpocapse ou vers des pommes et des poires. In : Entomologie appliquée à l'agriculture (AS Balachowsky, ed), tome II, vol 1, Masson, Paris, 653-674

Buès R, Toubon JF (1992) Polymorphisme enzymatique dans différentes populations de Cydia pomonella L (Lep Tortricidae). Acta CEcologica 13, 583-591

El Gamil FM, Gaaboub IA, EI Sawaf SK (1978) Biology of the codling moth Cydia pomonella $\mathrm{L}$ and effect of fruit age on its diapause induction. J Agric Sci Camb 90, 233-236

Feder JL, Chilcote CA, Bush GL (1988) Genetic differentiation between sympatric host races of apple maggot fly Rhagoletis pomonella. Nature 336, 61-64

Nei M (1978) Estimation of average heterozygosity and genetic distance from a small number of individuals. Genetics 89, 583-590

Pashley DP (1985) Genetic population structure of migratory moth: the Fall Armyworm (Lep Noctuidae). Ann Entomol Soc Am 78, 756-762

Phillips $\mathrm{PH}$, Barnes MM (1975) Host race formation among sympatric apple walnut and plum populations of codling moth, Laspeyresia pomonella. Ann Entomol Soc Am 68, 1053-1060

Pitcairn MJ, Zalom FG, Rice RE (1992) Degree-day forescasting of generation time of Cydia pomonella (Lep Tortricidae) populations in California. Environ Entomol 21, 441-446

Poitout S, Buès B (1974) Élevage de chenilles de 28 espèces de lépidoptères Noctuidae et de 2 espèces d'Arctiidae sur milieu artificiel simple, particularité de l'élevage selon les espèces. Ann Zool Ecol Anim $6,431-441$
Rield H, Croft BA (1978) The effects of photoperiod and effective temperatures on the seasonal phenology of the codling moth (Lep Tortricidae). Can Ent $110,455-470$

Sauphanor B, Benoit Magali, Bouvier JC, Perron G, Malezieux S, Frémond JC (1994) Un cas de résistance du carpocapse des pommes au diflubenzuron dans le sud-est de la France. Phytoma 458, 4649

Scherrer B (1984) Biostatistique. Gaëtan Morin, Québec, Canada, $850 \mathrm{p}$

Shel'Deshova GG (1967) Ecological factors determining distribution of the codling moth Laspeyresia pomonella $\mathrm{L}$ in the northern and southern hemispheres. Entomol Rev 46, 349-361

Slatkin M (1985) Rare alleles as indicators of gene flow. Evolution 39, 53-65

Slatkin M (1987) Gene flow and the geographic structure of natural populations. Science 236, 787-792

Sokal RR, Rohlf FJ (1981) Biometry. Freeman, San Francisco, $859 \mathrm{p}$

Steinberg S, Podoler H, Appelbaum SW (1992) Diapause induction in the codling moth, Cydia pomonella: effect of prediapause temperatures. Entom/ Exp App/62, 131-137

Swofford DL, Selander RB (1981) BIOSYS 1, a Fortran program for the comprehensive analysis of electrophoretic data in population genetics and systematics. J Hered 72, 281-283

Weir BS (1990) Intraspecific differenciation. In : Molecular Systematic (DM Hillis, C Moritz, eds), Sinauer Associates Inc, Sunderland, MA, USA, 373410

Wright S (1951) The genetical structure of populations. Ann Eugen 15, 323-354 